\title{
The efficacy of bleb needling revision with 5-fluorouracil in encapsulated bleb after unsuccessful trabeculectomy
}

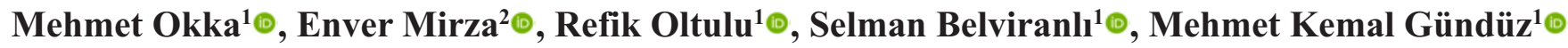 \\ ${ }^{1}$ Department of Ophthalmology, Necmettin Erbakan University, Meram School of Medicine, Konya, Turkey \\ ${ }^{2}$ Department of Ophthalmology, University of Health Sciences, Konya Training and Research Hospital, Konya, Turkey
}

DOI: $10.18621 /$ eurj.425740

\begin{abstract}
Objectives: To investigate the efficacy of the bleb needling revision (BNR) procedure with the adjunctive use of 5-Fluorouracil (5-FU) in encapsulated bleb after unsuccessful trabeculectomy.

Methods: We reviewed 16 eyes of 15 subjects who underwent BNR procedure due to encapsulated bleb after unsuccessful trabeculectomy. Demographic data, type of glaucoma, intraocular pressure (IOP) values of preBNR, IOP values of post-BNR at first day, first week and first month, follow up time and complications were recorded from patients' files, retrospectively.

Results: The mean follow up time was $53.1 \pm 26.4$ weeks. The average time between previous unsuccessful trabeculectomy and BNR was $11.12 \pm 8.79$ weeks. The mean IOP of pre-BNR was $26.0 \pm 4.4 \mathrm{mmHg}$ and significantly decreased to $12.4 \pm 5.0 \mathrm{mmHg}$ post-BNR at the first day $(p<0.001)$. The mean IOP values of post-BNR at the first week was $13.3 \pm 4.9 \mathrm{mmHg}$ and at the first month was $14.8 \pm 4.8 \mathrm{mmHg}$. According to the mean IOP of pre-BNR, the mean IOP values of post-BNR at the first week and at the first month were significantly lower $(p<0.001$ and $p<0.001$, respectively). Seven (44\%) eyes were achieved success and eight $(50 \%)$ eyes were achieved qualified success. One $(6 \%)$ eye was classified as the failure.

Conclusion: The bleb needling revision procedure with the adjunctive use of 5-FU in encapsulated bleb after unsuccessful trabeculectomy is a simple, useful and repeatable method to restore the dysfunctional bleb.
\end{abstract}

Keywords: Bleb needling revision, 5-Fluorouracil, encapsulated bleb, trabeculectomy, filtration surgery

Received: May 21, 2018; Accepted: January 22, 2019; Published Online: June 30, 2019

$\mathrm{T}$ rabeculectomy remains the gold standard surgical procedure in cases that intraocular pressure (IOP) couldn't control with antiglaucomatous medication [1]. Trabeculectomy is a very effective procedure to reduce IOP at the early postoperative period. Nevertheless, its efficiency may decreases over time for some complications such as dysfunctional blebs. Localization of the bleb, episcleral fibrosis, and bleb encapsulation are the bleb-related reasons for the failure of primary filtration surgery [2].
Encapsulated bleb usually seen within 2-8 weeks after filtration surgery and the incidence is reported between $2.5 \%$ and $29 \%$ in previous studies [3, 4]. Encapsulated bleb was characterized as an elevated, smooth surfaced, tense, thickened, dome-shaped fibrotic membrane. The mechanism of development of encapsulated bleb is not well understood and many factors are responsible for its formation. The encapsulated bleb wall that limiting aqueous outflow is composed of a fibrous, collagenous connective tissue

Address for correspondence: Enver Mirza, MD., University of Health Sciences, Konya Training and Research Hospital, Department of Ophthalmology, Konya, Turkey, E-mail: envermirza@gmail.com 
which is histopathologically produced as a result of fibroblastic proliferation [5]. Hence, inflammatory reaction and fibroblastic activity may increase the risk of developing encapsulated bleb [6].

The method of Bleb Needling Revision (BNR) which was first described by Ferrer in 1941, is now frequently preferred after unsuccessful filtration surgery [7]. The adjunctive use of antifibrotic agents such as 5-fluorouracil (5-FU) and mitomycin-C (MMC) in BNR is suggested to increase the rate of success of the procedure. 5-FU and MMC inhibit the scarring tissue formation and the proliferation of the fibroblasts which are the primary reasons for dysfunction of the blebs. Thus, BNR with antifibrotic agent is a simple and reliable method that can be an alternative treatment after dysfunctional blebs and reduce the IOP without causing additional healthy conjunctival surface loss and prevents patients from another glaucoma surgery. BNR has very good results but many factors such as age, the number of antiglaucomatous drug used before, history of previous ocular surgery, type of glaucoma, use of antimetabolite in primary filtration surgery and bleb morphology may affect the success of the procedure.

In addition, MMC has higher potency according to 5-FU but more complications have been reported due to use of MMC $[1,6]$. Therefore, we prefer to perform the procedure with 5-FU. In this study, we aimed to evaluate the efficiency of the BNR procedure in patients who underwent BNR with adjunctive use of 5FU after unsuccessful trabeculectomy.

\section{METHODS}

Sixteen eyes of 15 subjects who underwent BNR due to encapsulated bleb after unsuccessful trabeculectomy (IOP $\geq 21 \mathrm{mmHg}$ ) in Necmettin Erbakan University, Meram School of Medicine, Department of Ophthalmology between March 2015March 2017 were evaluated retrospectively. The study was approved by the local ethics committee (No:2018/1156) and followed the tenets of the Declaration of Helsinki. Demographic data, type of glaucoma, IOP values of pre-BNR, IOP values of postBNR at the first day, the first week and the first month which were measured by Goldmann applanation tonometry, follow up time and complications were recorded from patients files.

The BNR procedure was performed in the operation room using a 27-gauge needle with adjunctive use of 5-FU by two surgeons (MO and EM). After topical anesthetic (Proparacaine Hydrochloride $0.5 \%$ ), 5\% povidone-iodine solutions were instilled into the eye. A 27-gauge needle was inserted into the subconjunctival space to the tight Tenon's capsule around the bleb on the opposite site of the scleral flap, puncture the adherent tissue and lift the scleral flap. The flap was lifted with gentle sideto-side movement, breaking episcleral adhesions until the bleb is reformed. If simple flap dissection failed to form a nonsatisfactory bleb, the needle can be passed under the scleral flap into the anterior chamber. Then, the needle was removed and $5 \mathrm{mg}(0.2 \mathrm{ml}$ of $25 \mathrm{mg} / \mathrm{ml}$ solution) of 5-FU was subconjunctivally injected superior to the bleb. At the end of the procedure, antibiotic drops (moxifloxacin $0.5 \%$ ) were instilled into the eye.

History of previous unsuccessful trabeculectomy surgery with or without antifibrotic agents, the presence of dysfunctional blebs due to encapsulated bleb, the presence of patent iridectomy were the inclusion criterion. History of previous multiple trabeculectomies or filtration surgery other than trabeculectomy, the presence of non-patent iridectomy, the presence of corneal epithelial complications, and leaky blebs were the exclusion criterion. The BNR procedure was considered as success IOP $<21 \mathrm{mmHg}$ without antiglaucomatous medication; qualified success defined as IOP $<21 \mathrm{mmHg}$ with antiglaucomatous medication and failure defined as $\mathrm{IOP} \geq 21 \mathrm{mmHg}$ with antiglaucomatous medication.

\section{Statistical Analysis}

SPSS for Windows (version 17; SPSS Inc, Chicago, Illinois, USA) were used for the analysis. The paired t-test was used to compare IOP. A P value $<0.05$ was considered as significant.

\section{RESULTS}

Sixteen eyes of 15 subjects were included in the study. Three (20\%) subjects were female, 12 (80\%) subjects were male. The age of subjects ranged 
Table 1. Demographic data and IOP values

\begin{tabular}{|c|c|c|c|c|c|c|c|}
\hline $\begin{array}{l}\text { Eye } \\
\text { No. }\end{array}$ & Gender & Age & Type of Glaucoma & $\begin{array}{c}\text { Pre-BNR } \\
\text { IOP } \\
(\mathrm{mmHg})\end{array}$ & $\begin{array}{c}\text { Post-BNR } \\
\text { IOP at } \\
\text { first day } \\
\text { (mmHg) }\end{array}$ & $\begin{array}{c}\text { Post-BNR } \\
\text { IOP at first } \\
\text { week } \\
(\mathrm{mmHg})\end{array}$ & $\begin{array}{c}\text { Post-BNR } \\
\text { IOP at first } \\
\text { month } \\
(\mathrm{mmHg})\end{array}$ \\
\hline 1 & M & 22 & Traumatic & 21.2 & 7 & 10 & 14 \\
\hline 2 & M & 33 & POAG & 23.4 & 8 & 16 & 14,2 \\
\hline 3 & $\mathrm{~F}$ & 57 & POAG & 27 & 6 & 6 & 12 \\
\hline 4 & $\mathrm{~F}$ & 73 & POAG & 25.8 & 17 & 16.8 & 15.6 \\
\hline 5 & M & 25 & POAG & 25,5 & 12 & 12.5 & 11.5 \\
\hline 6 & M & 25 & POAG & 30.3 & 15.1 & 10.1 & 13.1 \\
\hline 7 & M & 65 & POAG & 26.1 & 15.1 & 11.1 & 11.1 \\
\hline 8 & M & 69 & Pseudoexfoliation & 23.7 & 17.7 & 10.7 & 14.7 \\
\hline 9 & M & 54 & Uveitic & 25 & 5 & 7 & 8 \\
\hline 10 & M & 45 & Pseudoexfoliation & 25.8 & 12.8 & 10.1 & 10.4 \\
\hline 11 & $\mathrm{~F}$ & 59 & POAG & 22.6 & 16.6 & 16.6 & 15.6 \\
\hline 12 & M & 37 & POAG & 21.2 & 16.2 & 16.2 & 17.2 \\
\hline 13 & M & 57 & POAG & 22.2 & 13.4 & 17.6 & 15.8 \\
\hline 14 & M & 35 & Traumatic & 25.1 & 12.3 & 9.4 & 16.4 \\
\hline 15 & M & 18 & Traumatic & 36.9 & 9.9 & 19.3 & 17.3 \\
\hline 16 & M & 62 & Neovascular & 34.4 & 19.7 & 23.7 & 29.7 \\
\hline
\end{tabular}

$\mathrm{BNR}=$ bleb needling revision, $\mathrm{IOP}=$ intraocular pressure, $\mathrm{POAG}=$ primary open-angle glaucoma, $\mathrm{M}=$ male, $\mathrm{F}=$ female

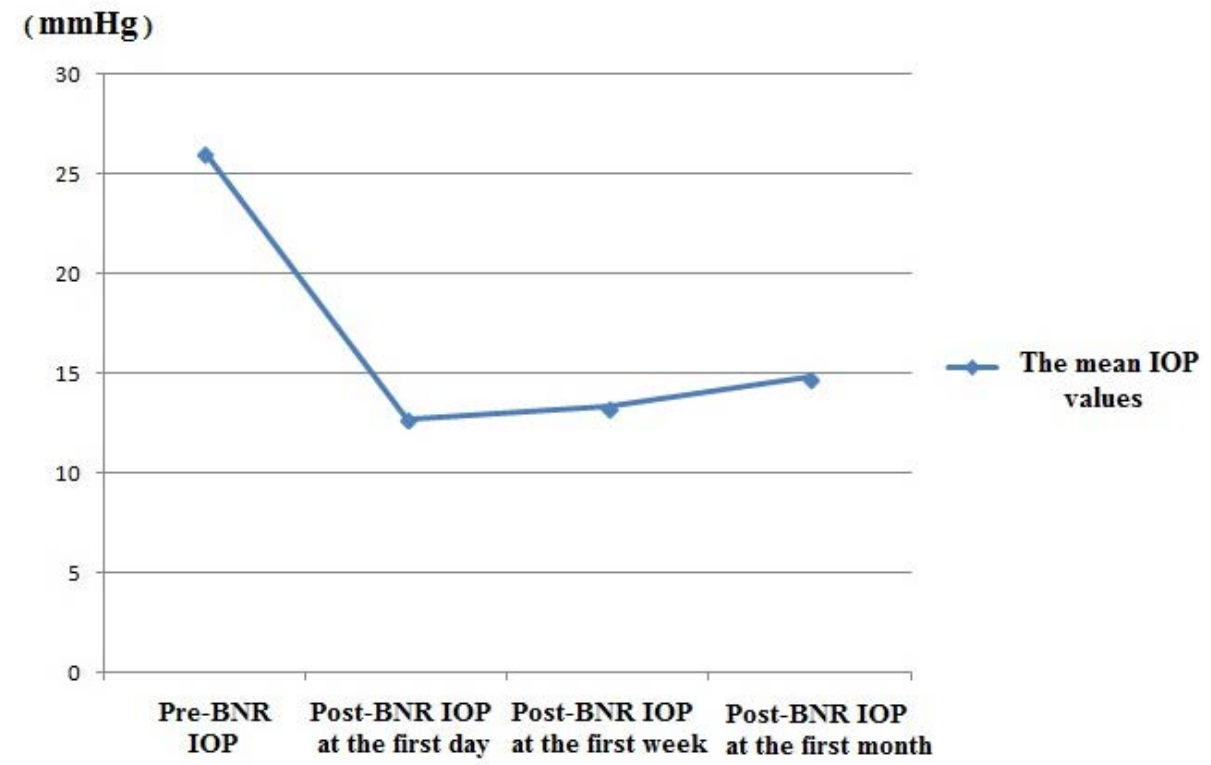

Figure 1. The mean IOP values of patients. $\mathrm{BNR}=$ bleb needling revision, $\mathrm{IOP}=$ intraocular pressur 
Table 2. Pre-BNR and Post-BNR characteristics

\begin{tabular}{ccccccc}
\hline $\begin{array}{c}\text { Eye } \\
\text { No. }\end{array}$ & $\begin{array}{c}\text { Time between } \\
\text { trabeculectomy } \\
\text { and BNR (week) }\end{array}$ & $\begin{array}{c}\text { Antimetabolite agent } \\
\text { use in previous } \\
\text { trabeculectomy }\end{array}$ & $\begin{array}{c}\text { Follow } \\
\text { up time } \\
\text { (week) }\end{array}$ & $\begin{array}{c}\text { The number of } \\
\text { antiglaucomatous } \\
\text { medication }\end{array}$ & Success & Complication \\
\hline 1 & 8 & $5-F U$ & 26 & None & Yes & None \\
2 & 4 & $5-F U$ & 80 & One & Qualified & None \\
3 & 4 & None & 124 & None & Yes & None \\
4 & 10 & None & 44 & One & Qualified & None \\
5 & 10 & $5-F U$ & 48 & None & Yes & None \\
6 & 7 & $5-F U$ & 36 & Two & Qualified & Late Leakage \\
7 & 9 & None & 25 & None & Yes & None \\
8 & 16 & None & 76 & One & Qualified & None \\
9 & 5 & None & 34 & None & Yes & None \\
10 & 29 & None & 61 & None & Yes & None \\
11 & 28 & None & 64 & Three & Qualified & Bullous Keratopathy \\
12 & 8 & None & 76 & Two & Qualified & Late Leakage \\
13 & 26 & $5-F U$ & 53 & One & Qualified & None \\
14 & 4 & $5-F U$ & 34 & None & Yes & None \\
15 & 6 & None & 40 & One & Qualified & None \\
16 & 4 & None & 28 & Three & Failure & None \\
\hline
\end{tabular}

$\mathrm{BNR}=$ bleb needling revision, $\mathrm{IOP}=$ intraocular pressure, 5 -FU $=5$-Fluorouracil

between 18-73 years old and the mean age of subjects was $46.0 \pm 18.2$ years (Table 1). All eyes were underwent conventional trabeculectomy previously and six of them were with adjunctive use of 5-FU (Table 2).

There were nine $(56 \%)$ eyes with primary openangle glaucoma (POAG) and seven (44\%) eyes with secondary glaucoma such as one eye had neovascular glaucoma, one eye had chronic uveitis, two eyes had pseudoexfoliation glaucoma, three eyes had glaucoma following serious trauma to the globe (Table 1). The average time between previous unsuccessful trabeculectomy and BNR ranged between 4-29 weeks and the mean time was $11.12 \pm 8.79$ weeks. The mean IOP of pre-BNR was $26.0 \pm 4.4 \mathrm{mmHg}$ and significantly decreased to $12.7 \pm 4.4 \mathrm{mmHg}$ post-BNR at the first day $(p<0.001)$. The mean IOP values of post-BNR at the first week were $13.3 \pm 4.9 \mathrm{mmHg}$ and at the first month was $14.8 \pm 4.8 \mathrm{mmHg}$ (Figure 1). The mean follow up time was $53.1 \pm 26.4$ weeks (range 25 to 124 weeks). According to the mean IOP of pre-BNR, the mean IOP values of post-BNR at the first week and at the first month were significantly lower ( $p<0.001$ and $p<0.001$, respectively). Seven (44\%) eyes were achieved success and eight (50\%) eyes were achieved qualified success. One $(6 \%)$ eye was classified as failure. Complications such as lateonset bleb leakage in two eyes and bullous keratopathy in one eye observed in three (19\%) eyes as shown in Table 2.

\section{DISCUSSION}

One of the most important factors that determining the success of trabeculectomy and bleb function is the degree of healing process in the filtration zone $[8,9]$. A variety of risk factors such as excessive surgical trauma and increased inflammatory process after an early period of filtration surgery which is related to the development of encapsulated bleb have been reported in the literature $[5,6]$. This suggests that these risk factors are not a definite etiology for the presence of encapsulated bleb formation [6]. Even so, surgical trauma and increased postoperative inflammation may probably lead to increase the risk of encapsulated bleb 
development [10]. Although there is currently no definitive treatment to prevent bleb failure and encapsulated bleb. Indeed, many drugs and different treatment methods are still being studied.

One of the drugs used to inhibit fibroblastic proliferation and enhance the success of the filtration surgery by allowing the bleb to remain functional is 5-FU. The use of 5-FU adjunctive with filtration surgery or BNR procedure and also injections after filtration surgery can be done. Many researchers have reported the efficacy of the BNR with 5-FU in unsuccessful bleb after filtration surgery $[11,12]$. Notably, IOP decreased by an average of $10.5 \mathrm{mmHg}$ (44.8\%) immediately after the BNR procedure in the study of Kapasi and Birt [11]. Broadway et al. [13] showed $47 \%$ success rate in encapsulated bleb after BNR with adjunctive 5-FU and it was reported that the mean IOP being reduced from $26.5 \mathrm{mmHg}$ to 15 $\mathrm{mmHg}$ after a median of $1 \mathrm{BNR}$ procedure. Also, the mean IOP was lower in the BNR with 5-FU group $(12.1 \pm 2.8 \mathrm{mmHg})$ compared to the medical treatment group $(15.1 \pm 2.1 \mathrm{mmHg})$ in the study of Suzuki and Susanna [14]. In our study, it was found that the mean IOP values decreased by $51 \%$ (reduced from $26.0 \pm$ $4.4 \mathrm{mmHg}$ to $12.7 \pm 4.4 \mathrm{mmHg}$ ) at the postoperative 1 st month compared to the mean preoperative IOP values.

MMC is another antifibrotic agent in filtration surgery and in BNR procedure but more complications have been reported due to use of MMC $[15,16]$. In a retrospective study comparing $\mathrm{MMC}$ and 5-FU, there was no difference in success between using MMC and 5-FU, and early postoperative IOP was reported to be the only predictor of success [17]. Complications such as hemorrhage, infection, late-onset bleb leakage, hypotonia, choroidal detachment and suprachoroidal hemorrhage can occur with rarely due to BNR procedure. Anand et al. [18] have reported that MMC is a more patent antifibrotic agent but also they reported that in the MMC group, blebitis and late bleb leakage were reported more frequently than in the 5FU group. In this study, we observed late-onset bleb leakage in two eyes and bullous keratopathy in one eye.

Previous studies have reported that the short time between previous unsuccessful trabeculectomy and the BNR procedure has an adverse effect on bleb function [19, 20]. Shetty et al. [21] suggested that immediate BNR after trabeculectomy could be unsuccessful due to the ongoing episcleral inflammation caused by the previous surgery. On the other hand, Gutierrez-Ortiz et al. [20] proposed that the success of the BNR procedure was highly correlated with glaucoma filtration surgery performed less than 4 months previously. In this study, the time elapsed between previous trabeculectomy and BNR procedure was ranged 4 to 29 weeks and additionally in twelve eyes the time was $\leq 10$ weeks. Indeed, we achieved success in seven of sixteen eyes and in eight eyes antiglaucomatous medication is needed after the BNR procedure (qualified success). The overall success rate of the BNR procedure in the study was $94 \%$.

Moreover, there may be an eventual decrease in efficiency in the BNR procedure over time. IOP $>30$ $\mathrm{mmHg}$ before the procedure, high IOP after the procedure, lack of use antimetabolite in previous filtration surgery, type of glaucoma are the risk factors that affect the efficiency of the BNR procedure [22]. Generally, repeated BNR with adjunctive 5-FU provide revive of filtration in dysfunctional bleb and prevent other surgery [23]. Indeed, additional surgery may be needed in some cases. Patients with neovascular glaucoma or secondary glaucoma (uveitic glaucoma, traumatic glaucoma, pseudoexfoliation glaucoma, etc.) constitute a high-risk group. In our study, in one risky eye which had neovascular glaucoma IOP couldn't control with antiglaucomatous medication after the BNR procedure and Ahmed glaucoma valve implantation had to be performed.

\section{CONCLUSION}

In conclusion, despite limitations of this study such as small sample size, retrospective design, and the absence of the control group, the BNR procedure with adjunctive use of 5-FU effectively reduces IOP or decreases the number of antiglaucomatous drugs in patients with encapsulated bleb and it is a simple, effective and repeatable method for the failure of bleb filtration after trabeculectomy.

\section{Conflict of interest}

The authors disclosed no conflict of interest during the preparation or publication of this manuscript. 


\section{Financing}

The authors disclosed that they did not receive any grant during conduction or writing of this study.

\section{REFERENCES}

[1] Errico D, Scrimieri F, Riccardi R, Fedeli R, Iarossi G. Trabeculectomy with double low dose of mitomycin $\mathrm{C}$ - two years of follow-up. Clin Ophthalmol 2011;5:1679-86.

[2] Azuara-Blanco A, Katz LJ. Dysfunctional filtering blebs. Surv Ophthalmol 1998;43:93-126.

[3] Yarangümeli A, Köz OG, Kural G. Encapsulated blebs following primary standard trabeculectomy: course and treatment. J Glaucoma 2004;13:251-5.

[4] Schwartz AL, Van Veldhuisen PC, Gaasterland DE, Ederer F, Sullivan EK, Cyrlin MN. The Advanced Glaucoma Intervention Study (AGIS): 5. Encapsulated bleb after initial trabeculectomy. Am J Ophthalmol 1999;127:8-19.

[5] Van Buskirk EM. Cysts of Tenon's capsule following filtration surgery. Am J Ophthalmol 1982;94:522-7.

[6] Mandal AK. Results of medical management and mitomycin $\mathrm{C}$-augmented excisional bleb revision for encapsulated filtering blebs. Ophthalmic Surg Lasers 1999;30:276-84.

[7] Ferrer H. Conjunctival dialysis in the treatment of glaucoma recurrent after sclerectomy. Am J Ophthalmol 1941;24:788-90.

[8] Addicks EM, Quigley HA, Green WR, Robin AL. Histologic characteristics of filtreing blebs in glaucomatous eyes. Arch Ophthalmol 1983;101:795-8.

[9] Skuta GL, Parrish RK: II. Wound healing in glaucoma filtration surgery. Surv Ophthalmol 1987;32:149-70.

[10] Ophir A. Encapsulated filtering bleb. A selective review-new deductions. Eye 1992;6:348-52.

[11] Kapasi MS, Birt CM. The efficacy of 5-fluorouracil bleb needling performed 1 year or more posttrabeculectomy: a retrospective study. J Glaucoma 2009;18:144-8.

[12] Paris G, Zhao M, Sponsel WE. Operative revision of nonfunctioning filtering blebs with 5-fluorouracil to regain intraocular pressure control. Clin Experiment Ophthalmol
2004;32:378-82.

[13] Broadway DC, Bloom PA, Bunce C, Thiagarajan M, Khaw PT. Needle revision of failing and failed trabeculectomy blebs with adjunctive 5-fluorouracil: survival analysis. Ophthalmology 2004;111:665-73.

[14] Suzuki R, Susanna R Jr. Early transconjunctival needling revision with 5-fluorouracil versus medical treatment in encapsulated blebs: a 12-month prospective study. Clinics (sao Paulo) 2013;68:1376-9.

[15] Panarelli JF, Vinod K, Huang G, Sidoti PA. Transconjunctival revision with mitomycin-C following failed trabeculectomy. J Glaucoma 2016;25:618-22.

[16] Mardelli PG, Lederer CM Jr, Murray PL, Pastor SA, Hassanein KM. Slit-lamp needle revision of failed filtering blebs using mitomycin C. Ophthalmology 1996;103:1946-55.

[17] Palejwala N, Ichhpujani P, Fakhraie G, Myers JS, Moster MR, Katz LJ. Single needle revision of failing filtration blebs: a retrospective comparative case series with 5-fluorouracil and mitomycin C. Eur J Ophthalmol 2010;20:1026-34.

[18] Anand N, Khan AJ. Glaucoma. Long-term outcomes of needle revision of trabeculectomy blebs with mitomycin $\mathrm{C}$ and 5-fluorouracil: a comparative safety and efficacy report. J Glaucoma 2009;18:513-20.

[19] Iwach AG, Delgado MF, Novack GD, Nguyen N, Wong PC. Transconjunctival mitomycin-C in needle revisions of filtering blebs. Ophthalmology 2003;110:734-42.

[20] Gutierrez-Ortiz C, Cabarga C, Teus MA. Prospective evaluation of preoperative factors associated with successful mitomycin $\mathrm{C}$ needling of failed filtration blebs. J Glaucoma 2006;15:98-102.

[21] Shetty RK, Wartluft L, Moster MR. Slit-lamp needle revision of failed fi ltering blebs using high-dose mitomycin $\mathrm{C}$. J Glaucoma 2005;14:52-6.

[22] Shin DH, Kim YY, Ginde SY, Kim PH, Eliassi-Rad B, Khatana $\mathrm{AK}$, et al. Risk factors for failure of 5-fluorouracil needling revision for failed conjunctival filtration blebs. Am J Ophthalmol 2001;132:875-80.

[23] Rashad MA. Efficacy of repeated 5-fluorouracil needling for failing and failed filtering surgeries based on simple gonioscopic examination. Clin Ophthalmol 2013;7:15-22. 